PROCEEDINGS OF THE

AMERICAN MATHEMATICAL SOCIETY

Volume 134, Number 12, December 2006, Pages 3653-3663

S 0002-9939(06)08401-2

Article electronically published on May 18, 2006

\title{
ON THE DENSITY OF BANACH SPACES $C(K)$ WITH THE GROTHENDIECK PROPERTY
}

\author{
CHRISTINA BRECH
}

(Communicated by Carl G. Jockusch, Jr.)

\begin{abstract}
Using the method of forcing we prove that consistently there is a Banach space of continuous functions on a compact Hausdorff space with the Grothendieck property and with density less than the continuum. It follows that the classical result stating that "no nontrivial complemented subspace of a Grothendieck space $C(K)$ is separable" cannot be strengthened by replacing "is separable" by "has density less than that of $l_{\infty}$ ", without using an additional set-theoretic assumption. Such a strengthening was proved by Haydon, Levy and Odell, assuming Martin's axiom and the negation of the continuum hypothesis. Moreover, our example shows that certain separation properties of Boolean algebras are quite far from the Grothendieck property.
\end{abstract}

\section{INTRODUCTION}

For an infinite compact Hausdorff space $K$, let $C(K)$ be the Banach space of the continuous real-valued functions on $K$, with the supremum norm. The purpose of this work is to show that the existence of a Grothendieck space $C(K)$ with density less than the continuum (denoted by $\mathfrak{c}$ ) is independent from the usual axioms of set theory. Recall that a Banach space $X$ is said to be a Grothendieck space (see 4 for more details) whenever each weak* convergent sequence in its dual $X^{*}$ converges weakly. To obtain this independence result we make the following two assertions:

$$
\begin{gathered}
\text { If } \mathfrak{p}=\mathfrak{c}, \text { then every Grothendieck space } C(K) \text { has density } \geq \mathfrak{c} . \\
\text { In a model obtained by forcing, there is a Grothendieck } \\
\text { space } C(K) \text { with density }<\mathfrak{c} .
\end{gathered}
$$

The main purpose of this work is to prove (II), since (I) is already known: it follows from a result of 9]. To present a direct proof of (I) here, we define the cardinal $\mathfrak{p}$ : we call $\mathfrak{p}$ the least infinite cardinal $\kappa$ for which there exists $\left(M_{\alpha}\right)_{\alpha<\kappa} \subseteq \wp(\mathbb{N})$ such that $\bigcap_{\alpha \in F} M_{\alpha}$ is infinite for all finite subsets $F$ of $\kappa$ and there is no infinite $M \subseteq \mathbb{N}$ such that $\left|M \backslash M_{\alpha}\right|<\infty$ for all $\alpha<\kappa$. This means that we can in some way diagonalize less than $\mathfrak{p}$ subsets of $\mathbb{N}$ which are finitely compatible. It is known that $\omega_{1} \leq \mathfrak{p} \leq \mathfrak{c}\left(\omega_{1}\right.$ is the first uncountable cardinal) and that MA (Martin's

Received by the editors September 26, 2004 and, in revised form, June 14, 2005.

2000 Mathematics Subject Classification. Primary 03E35; Secondary 46B20.

The author was supported by a scholarship from FAPESP (02/04531-6). This paper is part of the author's M.A. thesis at the University of São Paulo, under the guidance of Professor Piotr Koszmider. The author thanks him for his guidance and assistance during the preparation of this paper.

(C)2006 American Mathematical Society Reverts to public domain 28 years from publication 
axiom) implies that $\mathfrak{p}=\mathfrak{c}$. We have that every infinite compact Hausdorff space with weight less than $\mathfrak{p}$ has a nontrivial convergent sequence (see 6], Proposition $24 \mathrm{~A}$ ), and therefore $C(K)$ is not a Grothendieck space (see the proof of Theorem 9.5 of [11]). So, assuming $\mathfrak{p}=\mathfrak{c}$ we have that if $C(K)$ is a Grothendieck space, then $K$ has weight at least $\mathfrak{c}$, and by the Stone-Weierstrass Theorem, $C(K)$ has density at least $\mathfrak{c}$.

It follows from a result of 4 that no nontrivial complemented subspace of a Grothendieck space $C(K)$ is separable. A strengthening of this statement follows from a result of $[9$, assuming MA (or simply $\mathfrak{p}=\mathfrak{c}$ ) and the negation of $\mathrm{CH}$ (continuum hypothesis): each nontrivial complemented subspace of a nonreflexive Grothendieck space (hence each nontrivial complemented subspace of a Grothendieck space $C(K)$ ) has density at least $\mathfrak{c}$. Our result shows that we need an additional set-theoretic assumption to prove such strengthening.

Pełczyński asked (see [8]) if every Banach space of continuous functions should contain either a complemented copy of $c_{0}$ or a (complemented) copy of $l_{\infty}$. Talagrand (assuming $\mathrm{CH}$, see [16]) and Haydon (without any additional hypothesis; see [8]) answered this question negatively. Moreover, the space constructed by Talagrand does not have a quotient space isomorphic to $l_{\infty}$. On the other hand, Haydon, Levy and Odell proved in [9] that $\mathfrak{p}=\mathfrak{c}$ and the negation of $\mathrm{CH}$ imply that every Grothendieck space $C(K)$ has $l_{\infty}$ as a quotient. Our space has stronger properties than that constructed by Talagrand: it is a Grothendieck space $C(K)$ or, equivalently, a Banach space $C(K)$ with no complemented copies of $c_{0}$ (by a result of [13]) with density less than $\mathfrak{c}$, which is the density of $l_{\infty}$ (and therefore it has no quotient isomorphic to $\left.l_{\infty}\right)$.

Turning to properties of Boolean algebras, we would like to note that there are many of them which imply that $C(K)$ has the Grothendieck property, with $K$ its Stone space. Some of them are the subsequential completeness property (see [8]), subsequential interpolation property (see [7]), etc. However, all of them also imply that the Boolean algebra has cardinality at least $\mathfrak{c}$, which is not the case of ours. So, our space is a Grothendieck space $C(K)$, for $K$ the Stone space of a Boolean algebra, which does not have such properties. This illustrates that these properties are quite far from the Grothendieck property.

To show (II) we will not make use of well-known axioms like $\mathrm{CH}$ or $\mathfrak{p}=\mathfrak{c}$, as occur in the results of Talagrand and Haydon, Levy and Odell. Instead, we shall prove the consistency directly by forcing 1 Using a product of Sacks forcings (also known as the perfect set forcing) we obtain the model in which there is a Grothendieck space $C(K)$ with density less than $\mathfrak{c}$. It would be interesting to decide if axioms such as $\mathfrak{p}<\mathfrak{c}$ imply (II) directly. Other applications of the Sacks forcing in analysis can be found in 2] and [15].

The idea of showing (II) was motivated by a result of [10. In this work, Just and Koszmider showed that a certain compact Hausdorff space $K$ with weight less than $\mathfrak{c}$ (and so, $C(K)$ has density less than $\mathfrak{c}$ ) has no nontrivial convergent sequences. Although this is not sufficient for $C(K)$ to be a Grothendieck space, it is necessary. Thus, generalizing and modifying the methods used in [10] we prove that $C(K)$ is a Grothendieck space. Moreover, Schachermayer proved (see [13]) that a necessary (but not sufficient) condition for a Boolean algebra to have the

${ }^{1}$ A classical example of the use of forcing to obtain a result in analysis is the proof of the consistency of the automatic continuity of homomorphisms between Banach algebras (see [3]). 
Grothendieck property (that is, for $C(K)$ to be a Grothendieck space, where $K$ is its Stone space) is that it is not a countable union of a strictly increasing sequence of subalgebras. One of the results of [10] (which follows, also combining ours and that of [13]) is that our Boolean algebra is not such a union.

In this paper, $\mathcal{B}$ denotes an infinite Boolean algebra and $S(\mathcal{B})$ its Stone space. We use $\bigvee_{i \in I} b_{i}$ for the supremum of the family $\left(b_{i}: i \in I\right) \subseteq \mathcal{B}$, if it exists, and we say that a family $A \subseteq \mathcal{B}$ is an antichain in $\mathcal{B}$ if for each $a, b \in A$ with $a \neq b$ we have that $a \cdot b=0$. We denote by $\mu$ a real-valued finitely additive measure on $\mathcal{B}$, and if $\mu$ is bounded, $|\mu|$ denotes the variation of $\mu$. K will always denote an infinite compact Hausdorff space and $\operatorname{Bor}(K)$ the $\sigma$-algebra of its Borel sets. A Radon measure $\mu$ on $K$ is a real-valued $\sigma$-additive bounded regular measure on $\operatorname{Bor}(K)$, and $\|\mu\|$ denotes its norm (see [13] and 14] for the definitions).

Let $\mathcal{B}$ be a Boolean algebra. In what follows we will identify the Boolean algebra $\mathcal{B}$ with the Boolean algebra $C \operatorname{lop}(S(\mathcal{B}))$ of the closed and open subsets of $S(\mathcal{B})$, using the Stone duality. Recall that given a Radon measure $\mu$ on $K,\left.\mu\right|_{\mathcal{B}}$ is a finitely additive measure on $\mathcal{B}$. On the other hand, if $\mu$ is a finitely additive measure on $\mathcal{B}$, then there is a unique Radon measure $\tilde{\mu}$ on $K$ such that $\left.\tilde{\mu}\right|_{\mathcal{B}}=\mu$. Thus, we have a correspondence between finitely additive measures on $\mathcal{B}$ and Radon measures on $K$, and we will identify them in the sequel. Recall that the Riesz Representation Theorem guarantees that $C(S(\mathcal{B}))^{*}$ (the dual space of $C(S(\mathcal{B}))$ ) is isometric to the space of the Radon measures on $S(\mathcal{B})$. Hence we also identify each Radon measure on $S(\mathcal{B})$ (and thus, each finitely additive measure on $\mathcal{B}$ ) with the correspondent functional in $C(S(\mathcal{B}))^{*}$.

We use a standard terminology for the Sacks forcing: we denote by $\mathbb{S}$ the Sacks forcing and given $s \in \mathbb{S}$ and $p \in s$, let $s \mid p=\{q \in s: q \subseteq p$ or $p \subseteq q\} \in \mathbb{S}$. We denote by $l(n, s)$ the $n$th forking level of $s \in \mathbb{S}$, and we say that $s \leq_{n} t$ if $s \leq t$ and $l(n, s)=l(n, t)$ (see [1] for the definitions).

Given a regular cardinal $\kappa$, we denote by $\mathbb{S}^{\kappa}$ the product of $\kappa$ Sacks forcings, and given $s \in \mathbb{S}^{\kappa}$, a finite subset $F$ of $\operatorname{dom}(s)$ and $n \in \mathbb{N}$, we denote by $l(F, n, s)$ the set $\{\sigma: \operatorname{dom}(\sigma)=F$ and for all $\alpha \in F, \sigma(\alpha) \in l(n, s(\alpha))\}$. We say that $s \leq_{F, n} t$ if $s \leq t$ and $l(F, n, s)=l(F, n, t)$. Finally, if $s \in \mathbb{S}^{\kappa}$, if $F$ is a finite subset of $\operatorname{dom}(s)$ and if $\sigma$ is a function with domain $F$ such that $\sigma(\alpha) \in s(\alpha)$ for all $\alpha \in F$, let $s \mid \sigma \in \mathbb{S}^{\kappa}$ be such that $(s \mid \sigma)(\alpha)=s(\alpha)$ for $\alpha \in \operatorname{dom}(s) \backslash F$ and $(s \mid \sigma)(\alpha)=s(\alpha) \mid \sigma(\alpha)$ for $\alpha \in F$. We will need some results about this forcing, which are all proved in 1 .

In Section 2 we present some combinatorial results needed for the proof of (II) and in Section 3 we present the proof of the main result. The notation and terminology used are those of [5] for Grothendieck spaces and those of [1] for Sacks forcing.

\section{Some COMbinatorial RESUlts}

In this section we present some combinatorial results, which will be necessary in the proof of the main theorem. The following lemma is implicit in [12].

Lemma 2.1. Let $\mathcal{B}$ be a Boolean algebra and let $\kappa$ be an uncountable cardinal. Let $\left(a_{n}: n \in \mathbb{N}\right)$ be an antichain in $\mathcal{B}$ and let $\left(\mu_{k}: k \in \mathbb{N}\right)$ be a sequence in $C(S(\mathcal{B}))^{*}$. If $\left(N_{\xi}: \xi<\kappa\right)$ is an almost disjoint family in $\wp(\mathbb{N})$ (that is, a family of infinite subsets of $\mathbb{N}$ such that for all $\xi<\xi^{\prime}<\kappa, N_{\xi} \cap N_{\xi^{\prime}}$ is finite), then for all but countably many $\xi$ 's we have that for all $k \in \mathbb{N}$ and all $M \subseteq N_{\xi}$, if $\bigvee_{n \in M} a_{n}$ exists, 
then

$$
\mu_{k}\left(\bigvee_{n \in M} a_{n}\right)=\sum_{n \in M} \mu_{k}\left(a_{n}\right)
$$

Proof. Suppose that the lemma does not hold. Then there is an uncountable $X \subseteq \kappa$ such that for each $\xi \in X$ there is $k_{\xi} \in \mathbb{N}$ and an infinite set $M_{\xi} \subseteq N_{\xi}$ such that $\bigvee_{i \in M_{\xi}} a_{i}$ exists and $\sum_{i \in M_{\xi}} \mu_{k_{\xi}}\left(a_{i}\right) \neq \mu_{k_{\xi}}\left(\bigvee_{i \in M_{\xi}} a_{i}\right)$. We can assume without loss of generality that there are natural numbers $k$ and $m$ such that for all $\xi \in X$,

$$
\left|\mu_{k}\left(\bigvee_{i \in M_{\xi}} a_{i}\right)-\sum_{i \in M_{\xi}} \mu_{k}\left(a_{i}\right)\right|>\frac{1}{m}
$$

Fix $\xi \in X$. Let $\delta_{\xi}=\mu_{k}\left(\bigvee_{i \in M_{\xi}} a_{i}\right)-\sum_{i \in M_{\xi}} \mu_{k}\left(a_{i}\right)$. Since $\mu_{k}\left(\bigvee_{i \in M_{\xi}, i>l} a_{i}\right)$ converges to $\delta_{\xi}$ as $l \rightarrow \infty$, there are arbitrarily large $l \in \mathbb{N}$ such that

$$
\left|\mu_{k}\left(\bigvee_{i \in M_{\xi}, i>l} a_{i}\right)-\delta_{\xi}\right|<\left|\delta_{\xi}\right|-\frac{1}{m}
$$

Let $n$ be a natural number greater than $m \cdot\left\|\mu_{k}\right\|$ and let $\xi_{1}, \ldots, \xi_{n}$ be different ordinals in $X$ such that $\delta_{\xi_{j}}$ are either all positive or all negative. For each $1 \leq j \leq n$, let $l_{j}$ be a natural number as in $(*)$ and such that $\left(M_{\xi_{j}} \backslash\left\{0, \ldots, l_{j}\right\}\right)_{1 \leq j \leq n}$ are pairwise disjoint. Note that $(*)$ implies that $\mu_{k}\left(\bigvee_{i \in M_{\xi_{j}}, i>l_{j}} a_{i}\right)$ are also either all positive or all negative and that $\left|\mu_{k}\left(\bigvee_{i \in M_{\xi_{j}}, i>l_{j}} a_{i}\right)\right|>\frac{1}{m}$. Then,

$$
\left|\mu_{k}\left(\bigvee_{1 \leq j \leq n}\left(\bigvee_{i \in M_{\xi_{j}}, i>l_{j}} a_{i}\right)\right)\right|=\sum_{j=1}^{n}\left|\mu_{k}\left(\bigvee_{i \in M_{\xi_{j}}, i>l_{j}} a_{i}\right)\right| \geq n \cdot \frac{1}{m}>\left\|\mu_{k}\right\|,
$$

a contradiction. Therefore the lemma is true.

Lemma 2.2. Let $m, A$ and $N$ be natural numbers. For each $i<N$, let $G_{i}$ be a finite subset of $\mathbb{N}$ with cardinality at least $A+m$. Fix an infinite $X \subseteq \mathbb{N}$ and suppose that for each $i<N$ and each $k \in G_{i}, X_{k, i} \subseteq X$ is such that $X \subseteq \bigcup\left\{X_{k, i}: k \in F\right\}$ for all $i<N$ and all $F \subseteq G_{i}$ with $|F| \geq m$. Then, for each $i<N$, there is $H_{i} \subseteq G_{i}$ with cardinality at least $A$ such that $\bigcap\left\{X_{k, i}: i<N, k \in H_{i}\right\}$ is infinite.

Proof. Let $u$ be a nonprincipal ultrafilter in $\mathbb{N}$ which contains $X$. By the hypothesis, for each $i<N$, there are at most $m-1$ elements $k \in G_{i}$ for which $X_{k, i} \notin u$, since if they do not belong to $u$, their union does not belong to $u$ as well. Taking $H_{i}=\left\{k \in G_{i}: X_{k, i} \in u\right\}$, we have that $\left|H_{i}\right| \geq\left|G_{i}\right|-m \geq A$, which concludes the proof.

Lemma 2.3. Let $N$ be a natural number and for each $i<N$, let $G_{i} \subseteq \mathbb{N}$ be finite. For each $k \in \bigcup_{l<N} G_{l}$ and each $i, j<N$, let $\left(m_{i, j}^{k}(n): n \in G_{i}\right)$ be a sequence of positive real numbers such that

$$
\forall i, j<N, \quad \forall k \in \bigcup_{l<N} G_{l}, \quad \sum_{n \in G_{i}} m_{i, j}^{k}(n) \leq M,
$$

for a fixed positive real number $M$. Let $\eta$ be a positive real number and let $m$ be a natural number such that $m \cdot \eta>M$. Given a natural number $C$, there is a natural number $B$ depending on $C, N$ and $m$ such that if $\left|G_{i}\right| \geq B$ for all $i<N$, then, for each $i<N$, there is $G_{i}^{*} \subseteq G_{i}$ with cardinality $C$ such that for all $i, j<N$ with $i \neq j$, all $k \in G_{i}^{*}$ and all $n \in G_{j}^{*}$, we have that $m_{i, j}^{k}(n)<\eta$. 
Proof. We prove it by induction on $N$. If $N=1$, we are done.

So, assuming that the result holds for $N$, we prove it for $N+1$. Fix a natural number $C$ and let $C^{\prime}=C+C \cdot m$. By the inductive hypothesis, there is $B\left(N, C^{\prime}\right) \in \mathbb{N}$ satisfying the lemma for $N$ and $C^{\prime}$. We claim that

$$
B(N+1, C)=\max \left\{C+N^{2} \cdot C^{\prime} \cdot m, B\left(N, C^{\prime}\right)\right\}
$$

works.

Since $B(N+1, C) \geq B\left(N, C^{\prime}\right)$, we have that for each $i<N$, there is $G_{i}^{* *} \subseteq G_{i}$ with $\left|G_{i}^{* *}\right|=C^{\prime}$ satisfying the thesis. Taking $K=\bigcup\left\{G_{i}^{* *}: i<N\right\}$, we have that $|K| \leq N \cdot C^{\prime}$. For each $j<N$ and each $k \in K$, there at most $m$ elements $n$ of $G_{N}$ such that $m_{N, j}^{k}(n) \geq \eta$. So, taking

$$
G_{N}^{*}=\left\{n \in G_{N}: m_{N, j}^{k}(n)<\eta \text {, for all } k \in K \text { and all } j<N\right\},
$$

we have that $\left|G_{N}^{*}\right| \geq C$ and $m_{N, j}^{k}(n)<\eta$ for all $k \in K$, all $j<N$ and all $n \in G_{N}^{*}$. We can suppose without loss of generality that $\left|G_{N}^{*}\right|=C$. Since for each $i<N$ and each $k \in G_{N}^{*}$ there are at most $m$ elements $n$ of $G_{i}^{* *}$ such that $m_{i, N}^{k}(n) \geq \eta$, taking

$$
G_{i}^{*}=\left\{n \in G_{i}^{* *}: m_{i, N}^{k}(n)<\eta, \text { for all } k \in G_{N}^{*}\right\},
$$

we have that $\left|G_{i}^{*}\right| \geq C$ and for each $i, j<N$ with $i \neq j$, each $n \in G_{i}^{*}$ and each $k \in G_{j}^{*}$ we have that $m_{i, j}^{k}(n)<\eta$, which concludes the proof.

Proposition 2.4. Let $s \in \mathbb{S}^{\kappa}$, let $E \subseteq \mathbb{N}$ be finite and let $\varepsilon$ and $M$ be positive real numbers. Let $\left(\dot{\mu}_{k}: k \in \mathbb{N}\right)$ be a sequence of names for finitely additive measures on the Boolean algebra $\wp(\mathbb{N})$, let $\left(\dot{A}_{k}: k \in \mathbb{N}\right)$ be a sequence of names for subsets of $\mathbb{N}$ and let $\dot{X}$ be a name for a subset of $\mathbb{N}$. Suppose that

$$
s \Vdash\left\{\begin{array}{l}
\forall k \in \mathbb{N}, \quad\left\|\dot{\mu}_{k}\right\|<\check{M}, \\
\forall k, k^{\prime} \in \mathbb{N}, \quad k \neq k^{\prime}, \quad \dot{A}_{k} \cap \dot{A}_{k^{\prime}}=\emptyset, \\
\dot{X} \text { is infinite, } \\
\check{E} \cap \dot{X}=\emptyset, \\
\forall k \in \dot{X}, \quad\left|\dot{\mu}_{k}\left(\dot{A}_{k}\right)\right| \geq \check{\varepsilon} .
\end{array}\right.
$$

Given a natural number $N$, a finite subset $F$ of $\kappa$ and a positive real number $\delta$, there are: $s^{*} \in \mathbb{S}^{\kappa}$ with $s^{*} \leq_{F, N} s ; a^{*} \subseteq \mathbb{N} ; E^{*} \subseteq \mathbb{N}$ with $\left|E^{*}\right| \leq 2^{N|F|}$; a sequence of names $\left(\dot{A}_{k}^{*}: k \in \mathbb{N}\right)$ for subsets of $\mathbb{N}$; and a name $\dot{X}^{*}$ for a subset of $\mathbb{N}$ such that $s^{*}$ forces that:

(1) for all $k \in \mathbb{N}, \dot{A}_{k}^{*}=\dot{A}_{k} \backslash \check{a}^{*}$ and so, for all $k, k^{\prime} \in \mathbb{N}, k \neq k^{\prime}, \dot{A}_{k}^{*} \cap \dot{A}_{k^{\prime}}^{*}=\emptyset$;

(2) $\dot{X}^{*} \subseteq \dot{X}$ and $\dot{X}^{*}$ is infinite;

(3) for all $k \in \dot{X}^{*},\left|\dot{\mu}_{k}\right|\left(\check{a}^{*}\right) \leq \check{\delta}$ and so, for all $k \in \dot{X}^{*},\left|\dot{\mu}_{k}\left(\dot{A}_{k}^{*}\right)\right| \geq \check{\varepsilon}-\check{\delta}$;

(4) for all $k \in \check{E},\left|\dot{\mu}_{k}\right|\left(\check{a}^{*}\right) \leq \check{\delta}$;

(5) there is $k \in \check{E}^{*}$ such that $\left|\dot{\mu}_{k}\left(\check{a}^{*}\right)\right| \geq \check{\varepsilon}-\check{\delta}$;

(6) $\check{E}^{*} \subseteq \dot{X} \backslash \dot{X}^{*}$.

Proof. First, take $K=2^{N|F|}$ and $\eta=\frac{\delta}{K}$. Fix a natural number $m$ such that $m \cdot \eta>M$. Let $B \in \mathbb{N}$ be large enough (we need it large enough in order to have a number greater than 1 after several applications of Lemmas 2.2 and 2.3).

We will define $a^{*}$ as the "union of some $\dot{A}_{k}$ 's" with $k \in \dot{X}$. To find the $k$ 's that will work, we have to decide many (but finitely many) elements of $\dot{X}^{*}$, and after 
that, we will eliminate those which do not serve. So, take $L=l(F, N, s)$ and let

$D=\left\{p \in \mathbb{S}^{\kappa}\right.$ : there is $G \subseteq \mathbb{N}$ with $|G|=B$ and such that $\left.p \Vdash \check{G} \subseteq \dot{X}\right\}$.

Since $D$ is dense below $s$ and open, by Lemma 1.8 of [1] there is $s^{\prime} \leq_{F, N} s$ such that $s^{\prime} \mid \sigma \in D$ for all $\sigma \in L$. Hence for each $\sigma \in L$, there is $G_{\sigma} \subseteq \mathbb{N}$ with cardinality $B$ such that $s^{\prime} \mid \sigma$ forces that $\check{G}_{\sigma} \subseteq \dot{X}$.

Now, we want to decide $\dot{A}_{k}$ for each $k \in G_{\sigma}$ and each $\sigma \in L$. Let $G=\bigcup_{\sigma \in L} G_{\sigma}$ and

$$
D^{\prime}=\left\{p \in \mathbb{S}^{\kappa}: \text { for all } k \in G \text { there is } A_{k} \subseteq \mathbb{N} \text { such that } p \Vdash \check{A}_{k}=\dot{A}_{k}\right\} .
$$

Again, since $D^{\prime}$ is dense below $s^{\prime}$ and open, applying Lemma 1.8 of 1 we obtain $s^{\prime \prime} \leq_{F, N} s^{\prime}$ such that for each $\sigma \in L$ we have $s^{\prime \prime} \mid \sigma \in D^{\prime}$. Hence for each $\sigma \in L$ and each $k \in G$, there is $A_{k}(\sigma) \subseteq \mathbb{N}$ such that $s^{\prime \prime} \mid \sigma$ forces that $\check{A}_{k}(\sigma)=\dot{A}_{k}$ and therefore, for each $\sigma \in L,\left(A_{k}(\sigma)\right)_{k \in G}$ is pairwise disjoint.

Since we want the measures of $a^{*}$ to satisfy properties (3), (4) and (5), and the names $\dot{A}_{n}^{*}$ to satisfy properties (1) and (3), we will approximate the values of the measures $\dot{\mu}_{k}$ for $k \in E \cup G$ in the sets $A_{n}(\sigma)$ for $\sigma \in L$ and $n \in G_{\sigma}$.

Claim 1. For each $\sigma, \sigma^{\prime} \in L$, each $k \in E \cup G$ and each $n \in G_{\sigma}$, there is $m_{\sigma, \sigma^{\prime}}^{k}(n) \in \mathbb{R}$ and $t \leq_{F, N} s^{\prime \prime}$ such that for all $\sigma, \sigma^{\prime} \in L$, all $k \in E \cup G$ and all $n \in G_{\sigma}, t \mid \sigma$ forces that $\left|\dot{\mu}_{k}\right|\left(\check{A}_{n}\left(\sigma^{\prime}\right)\right) \leq \check{m}_{\sigma, \sigma^{\prime}}^{k}(n)$ and such that for all $\sigma, \sigma^{\prime} \in L$ and all $k \in E \cup G$,

$$
\sum_{n \in G_{\sigma}} m_{\sigma, \sigma^{\prime}}^{k}(n)<M
$$

Proof of Claim 1. Since $E \cup G$ is finite and $s$ forces that $\left\|\dot{\mu}_{k}\right\|<\check{M}$ for all $k \in \check{E} \cup \check{G}$, it also forces that there is $\theta>0$ such that $\check{M}-\left\|\dot{\mu}_{k}\right\| \geq \theta$ for all $k \in \check{E} \cup \check{G}$. Thus, there is $p \leq_{F, N} s^{\prime \prime}$ such that $p$ forces that $\check{M}-\left\|\dot{\mu}_{k}\right\| \geq \check{\theta}$ for all $k \in E \cup G$.

There is $t \leq_{F, N} p$ and for each $\sigma, \sigma^{\prime} \in L$, each $n \in G_{\sigma}$ and each $k \in E \cup G$, there is $m_{\sigma, \sigma^{\prime}}^{k}(n) \in \mathbb{R}$ such that

$$
t\left|\sigma \Vdash 0 \leq \check{m}_{\sigma, \sigma^{\prime}}^{k}(n)-\right| \dot{\mu}_{k} \mid\left(\check{A}_{n}\left(\sigma^{\prime}\right)\right)<\frac{\check{\theta}}{2\left|G_{\sigma}\right|} .
$$

Recall that for each $\sigma \in L,\left(A_{k}(\sigma)\right)_{k \in G}$ is pairwise disjoint. Therefore, for all $\sigma, \sigma^{\prime} \in L$ and all $k \in \check{E} \cup \check{G}, t \mid \sigma$ forces that

$$
\check{M}-\sum_{n \in G_{\sigma}} \check{m}_{\sigma, \sigma^{\prime}}^{k}(n) \geq \check{M}-\sum_{n \in G_{\sigma}}\left(\left|\check{\mu}_{k}\right|\left(\check{A}_{n}\left(\sigma^{\prime}\right)\right)-\frac{\check{\theta}}{2\left|G_{\sigma}\right|}\right) \geq \check{M}-\left\|\check{\mu}_{k}\right\|-\frac{\check{\theta}}{2} \geq \frac{\check{\theta}}{2}>0
$$

and so, $\sum_{n \in G_{\sigma}} m_{\sigma, \sigma^{\prime}}^{k}(n)<M$. Moreover, for each $n \in G_{\sigma}$, each $\sigma, \sigma^{\prime} \in L$ and each $k \in \check{E} \cup \check{G}, t \mid \sigma$ forces that $\left|\dot{\mu}_{k}\right|\left(\check{A}_{n}\left(\sigma^{\prime}\right)\right) \leq \check{m}_{\sigma, \sigma^{\prime}}^{k}(n)$, concluding the proof of the claim.

From the fact that for each $\sigma, \sigma^{\prime} \in L$ and each $k \in E, \sum_{n \in G_{\sigma}} m_{\sigma, \sigma^{\prime}}^{k}(n)<M$, it follows that for each $\sigma, \sigma^{\prime} \in L$ and each $k \in E$ there are at most $m$ elements $n$ of $G_{\sigma}$ such that $m_{\sigma, \sigma^{\prime}}^{k}(n) \geq \eta$. Since $B$ is large enough, we can assume without loss of generality that

$$
\forall \sigma, \sigma^{\prime} \in L, \quad \forall k \in E, \quad \forall n \in G_{\sigma}, \quad m_{\sigma, \sigma^{\prime}}^{k}(n)<\eta,
$$

and by Lemma 2.3, we can assume without loss of generality that

$$
\forall \sigma, \sigma^{\prime} \in L, \quad \sigma \neq \sigma^{\prime}, \quad \forall n \in G_{\sigma}, \quad \forall k \in G_{\sigma^{\prime}}, \quad m_{\sigma, \sigma^{\prime}}^{k}(n)<\eta .
$$


To obtain $\dot{X}^{*}$ satisfying (2), for each $\sigma \in L$ and each $n \in G_{\sigma}$, let $\dot{X}_{n, \sigma}$ be a name such that $t$ forces that $\dot{X}_{n, \sigma}=\left\{k \in \dot{X}:\left|\dot{\mu}_{k}\right|\left(\check{A}_{n}(\sigma)\right)<\eta\right\}$.

Claim 2. There is $s^{*} \leq_{F, N} t$ and for each $\sigma \in L$ there is a nonempty $H_{\sigma} \subseteq G_{\sigma}$ such that $s^{*}$ forces that $\bigcap\left\{\dot{X}_{n, \sigma}: n \in \check{H}_{\sigma}, \sigma \in L\right\}$ is infinite.

Proof of Claim 2. Let $\left(\sigma_{i}: i<K\right)$ be an enumeration of $L$. To prove the claim, we will proceed by induction on $j<K$ to construct a sequence of conditions $s^{j}$ such that $s^{j+1} \leq_{F, N} s^{j} \leq_{F, N} t$, and for each $\sigma \in L$, we construct a sequence of finite nonempty sets $H_{\sigma, j}$ with $H_{\sigma, j+1} \subseteq H_{\sigma, j} \subseteq G_{\sigma}$ such that $s^{j} \mid \sigma_{j}$ forces that $\bigcap\left\{\dot{X}_{n, \sigma}: n \in \check{H}_{\sigma, j}, \sigma \in L\right\}$ is infinite.

For the construction, fix $0 \leq j<K$ and suppose we already have $s^{j}$ and $H_{\sigma, j}$ for all $\sigma \in L$ as wanted. We have that $s^{j}$ forces that $\dot{X} \subseteq \bigcup\left\{\dot{X}_{n, \sigma}: n \in H\right\}$ for all $\sigma \in L$ and all $H \subseteq H_{\sigma, j}$ with $|H| \geq m$. For if not, then there would be $\sigma \in L$, $k \in \mathbb{N}, H \subseteq H_{\sigma, j}$ with $|H| \geq m$ and $\left(s^{j}\right)^{\prime} \leq s^{j}$ such that $\left(s^{j}\right)^{\prime}$ forces that

$$
k \in \dot{X} \backslash \bigcup\left\{\dot{X}_{n, \sigma}: n \in H\right\} \text {. }
$$

Since for each $\sigma \in L,\left(A_{n}(\sigma)\right)_{n \in H}$ are pairwise disjoint, we would have that $\left(s^{j}\right)^{\prime}$ forces that $\left|\dot{\mu}_{k}\right|\left(\bigcup\left\{\check{A}_{n}(\sigma): n \in H\right\}\right) \geq \sum_{n \in H}\left|\dot{\mu}_{k}\right|\left(\check{A}_{n}(\sigma)\right) \geq \check{m} \cdot \check{\eta}>\check{M}$, contradicting our hypothesis. We apply Lemma 2.2 in $V[G]$, and using Lemma 1.8 of [1] we have that there is $s^{j+1} \leq_{F, N} s^{j}$, and for each $\sigma \in L$ there is $H_{\sigma, j+1} \subseteq H_{\sigma, j}$, such that $s^{j+1} \mid \sigma_{j+1}$ forces that $\bigcap\left\{\dot{X}_{n, \sigma}: \sigma \in L, n \in \check{H}_{\sigma, j+1}\right\}$ is infinite. Since $C$ is large enough, we can assume each $H_{\sigma, K-1}$ to be nonempty, and taking $s^{*}=s^{K-1}$ and $H_{\sigma}=H_{\sigma, K-1}$ we conclude the proof of the claim.

For each $\sigma \in L$ we take $k_{\sigma} \in H_{\sigma}$ of Claim 2. We define $a^{*}=\bigcup_{\sigma \in L} A_{k_{\sigma}}(\sigma)$ and $E^{*}=\left\{k_{\sigma}: \sigma \in L\right\}$. For each $k \in \mathbb{N}$, we define $\dot{A}_{k}^{*}$ names for $\dot{A}_{k} \backslash a^{*}$ and $\dot{X}^{*}$ a name such that

$$
s^{*} \Vdash \dot{X}^{*}=\bigcap\left\{\dot{X}_{k, \sigma}: k \in \check{H}_{\sigma}, \sigma \in L\right\} \backslash \check{E}^{*} .
$$

Claim 3. $s^{*}$ forces that for all $k \in \bigcap\left\{\dot{X}_{n, \sigma^{\prime}}: n \in \check{H}_{\sigma^{\prime}}, \sigma^{\prime} \in L\right\}$ and all $\sigma \in L$, $\left|\dot{\mu}_{k}\right|\left(\check{A}_{k_{\sigma}}(\sigma)\right)<\check{\eta}$.

Proof of Claim 3. Suppose that $s^{*}$ forces that $k \in \dot{X}_{n, \sigma}$ for each $n \in H_{\sigma}$ and each $\sigma \in L$. Then, by the definition of $\dot{X}_{n, \sigma}$ it means that $s^{*}$ forces that $\left|\dot{\mu}_{k}\right|\left(\check{A}_{n}(\sigma)\right)<\check{\eta}$ for each $n \in H_{\sigma}$ and each $\sigma \in L$. Since each $k_{\sigma} \in H_{\sigma}$, we conclude the claim.

Let us now verify that we have everything we wanted: first, note that by the definition of $\dot{A}_{k}^{*}$ we have that $s^{*}$ forces that $\dot{A}_{k}^{*}=\dot{A}_{k} \backslash \check{a}^{*}$ and $\left(\dot{A}_{k}^{*}\right)_{k \in \mathbb{N}}$ are pairwise disjoint, since it forces that $\left(\dot{A}_{k}\right)_{k \in \mathbb{N}}$ are pairwise disjoint. Therefore we obtain (1).

By the definition of $\dot{X}^{*}$ we have that $s^{*}$ forces that $\dot{X}^{*} \subseteq \dot{X}$. By Claim 2 we have that $s^{*}$ forces that $\dot{X}^{*}$ is infinite, since $E^{*}$ is finite. So we obtain (2).

By Claim 3 we have that

$$
s^{*} \Vdash \forall k \in \dot{X}^{*}, \quad\left|\dot{\mu}_{k}\right|\left(\check{a}^{*}\right)=\left|\dot{\mu}_{k}\right|\left(\bigcup_{\sigma \in L} \check{A}_{k_{\sigma}}(\sigma)\right) \leq \sum_{\sigma \in L}\left|\dot{\mu}_{k}\right|\left(\check{A}_{k_{\sigma}}(\sigma)\right) \leq \check{K} \cdot \check{\eta} \leq \check{\delta} .
$$

By the hypothesis of the proposition, $s$ forces that $\left|\dot{\mu}_{k}\left(\dot{A}_{k}\right)\right| \geq \check{\varepsilon}$ for each $k \in \dot{X}$. To obtain (3), note that

$$
s^{*} \Vdash \forall k \in \dot{X}^{*}, \quad\left|\dot{\mu}_{k}\left(\dot{A}_{k}^{*}\right)\right| \geq\left|\dot{\mu}_{k}\left(\dot{A}_{k}\right)\right|-\left|\dot{\mu}_{k}\right|\left(\check{a}^{*}\right) \geq \check{\varepsilon}-\check{\delta} .
$$


To verify (4), note that Claim 1 and (7) imply that for all $\sigma, \sigma^{\prime} \in L$, all $k \in E$ and all $n \in G_{\sigma}$,

$$
s^{*}|\sigma \Vdash| \dot{\mu}_{k} \mid\left(\check{A}_{n}\left(\sigma^{\prime}\right)\right) \leq \check{m}_{\sigma, \sigma^{\prime}}^{k}(n)<\check{\eta} .
$$

Since for all $\sigma \in L, k_{\sigma} \in H_{\sigma} \subseteq G_{\sigma}$, then, for each $\sigma \in L$ and each $k \in E$ we have that

$$
s^{*}|\sigma \Vdash| \dot{\mu}_{k}\left|\left(\check{a}^{*}\right)=\right| \dot{\mu}_{k}\left|\left(\bigcup_{\sigma^{\prime} \in L} \check{A}_{k_{\sigma^{\prime}}}\left(\sigma^{\prime}\right)\right) \leq \sum_{\sigma^{\prime} \in L}\right| \dot{\mu}_{k} \mid\left(\check{A}_{k_{\sigma^{\prime}}}\left(\sigma^{\prime}\right)\right) \leq \check{K} \cdot \check{\eta} \leq \check{\delta},
$$

and so, by Lemma 1.9 of [1] we obtain (4).

To verify (5), note that Claim 1 and (8) imply that for all $\sigma, \sigma^{\prime} \in L$ with $\sigma \neq \sigma^{\prime}$, all $n \in G_{\sigma}$ and all $k \in G_{\sigma}^{\prime}$,

$$
s^{*}|\sigma \Vdash| \dot{\mu}_{k} \mid\left(\check{A}_{n}\left(\sigma^{\prime}\right)\right) \leq \check{m}_{\sigma, \sigma^{\prime}}^{k}(n)<\check{\eta} .
$$

Since for all $\sigma \in L, k_{\sigma} \in H_{\sigma} \subseteq G_{\sigma}$, we have that for each $\sigma \in L$,

$$
s^{*}|\sigma \Vdash| \dot{\mu}_{k_{\sigma}}\left(a^{*}\right)|\geq| \dot{\mu}_{k_{\sigma}}\left(\check{A}_{k_{\sigma}}(\sigma)\right)\left|-\sum_{\sigma^{\prime} \in L, \sigma^{\prime} \neq \sigma}\right| \dot{\mu}_{k_{\sigma}} \mid\left(\check{A}_{k_{\sigma^{\prime}}}\left(\sigma^{\prime}\right)\right)>\check{\varepsilon}-\check{K} \cdot \check{\eta}=\check{\varepsilon}-\check{\delta},
$$

and again by Lemma 1.9 of 1 we obtain (5).

By the definition of $E^{*}$ and that of $\dot{X}^{*}$ we have (6).

\section{The PRoOf OF THE MAIN THEOREM}

We now show how the main result follows from Proposition 2.4.

Theorem 3.1. Let $\kappa>\omega_{1}$ be a regular cardinal. Let $G$ be an $\mathbb{S}^{\kappa}$-generic filter over a set-theoretic universe $V$ where $C H$ holds. In $V[G]$, if $K$ is the Stone space of the Boolean algebra $\wp(N) \cap V$, then $C(K)$ is a Banach space with the Grothendieck property and density $\omega_{1}$ which is less than $\kappa=\mathfrak{c}$.

Proof. First we work in $V[G]$. By Theorem 1.11 of $[1],|\wp(\mathbb{N}) \cap V|=\omega_{1}$, and by Theorem 1.14 of $\left[1, \omega_{1}<\kappa=\mathfrak{c}\right.$. So, $K$ has weight $\omega_{1}$ and $C(K)$ has density $\omega_{1}$, which is less than $\mathfrak{c}$.

Now suppose that $C(K)$ is not a Grothendieck space. Then there is a sequence $\left(\mu_{k}\right)_{k \in \mathbb{N}} \subseteq C(K)^{*}$ which is weak* convergent to $\mu \in C(K)^{*}$ but does not converge weakly. If $\left\{\mu_{k}: k \in \mathbb{N}\right\}$ were weakly compact, by the Eberlein-Šmulian Theorem, it would be sequentially weakly compact. Then there would be infinite and disjoint sets $M_{1}, M_{2} \subseteq \mathbb{N}$ such that $\left(\mu_{k}\right)_{k \in M_{i}}$ is weakly convergent to $\nu_{i}$ for $i=1,2$, and $\nu_{1} \neq \nu_{2}$. Since weak convergence implies weak* convergence, we would have that $\left(\mu_{k}\right)_{k \in M_{i}}$ converges weakly* to $\nu_{i}$ for $i=1,2$, a contradiction.

So, we can assume that $\left\{\mu_{k}: k \in \mathbb{N}\right\}$ is not weakly compact. By the Uniform Boundedness Principle, $\left(\mu_{k}\right)_{k \in \mathbb{N}}$ is a bounded sequence. By the DieudonnéGrothendieck Theorem (Theorem VII.14 in [5]), there is a pairwise disjoint sequence $\left(U_{k}\right)_{k \in \mathbb{N}}$ of open subsets of $K$ and $\varepsilon>0$ such that for all $k_{0} \in \mathbb{N}$ there is $k \geq k_{0}$ and $n_{k} \in \mathbb{N}$ such that $\left|\mu_{n_{k}}\left(U_{k}\right)\right| \geq \varepsilon$. Since $K$ is a Boolean space, using the regularity of each $\mu_{k}$, we can assume without loss of generality that $U_{k}=B_{k}$ for some $B_{k} \in \mathcal{B}$. Moreover, if for some $k \in \mathbb{N}$ we have that $\left|\mu_{k}\left(B_{k_{i}}\right)\right| \geq \varepsilon$ for some sequence $\left(k_{i}\right)_{i \in \mathbb{N}} \subseteq \mathbb{N}$, it follows that $\left|\mu_{k}\right|\left(\bigcup_{i \in \mathbb{N}} B_{k_{i}}\right) \geq \sum_{i \in \mathbb{N}}\left|\mu_{k}\left(B_{k_{i}}\right)\right|=\infty$, contradicting the fact that $\mu_{k}$ is bounded. So, let $i_{0} \in \mathbb{N}$ and $n_{0} \in \mathbb{N}$ be such that $\left|\mu_{n_{0}}\left(B_{i_{0}}\right)\right| \geq \varepsilon$, and we construct by induction $i_{k+1}>i_{k}$ and $n_{k+1}>n_{k}$ such that $\left|\mu_{n_{k}}\left(B_{i_{k}}\right)\right| \geq \varepsilon$. Let $A_{k}=B_{i_{k}}$, and we have that $\left(A_{k}\right)_{k \in \mathbb{N}} \subseteq \wp(\mathbb{N}) \cap V$ are pairwise disjoint and $\left(n_{k}\right)_{k \in \mathbb{N}} \subseteq \mathbb{N}$ is an increasing sequence such that for all $k \in \mathbb{N},\left|\mu_{n_{k}}\left(A_{k}\right)\right| \geq \varepsilon$. 
Working now in $V$, let $\dot{\mu}_{k}$ be a name for the restriction of $\mu_{n_{k}}$ to the Boolean algebra $\wp(\mathbb{N}) \cap V$. Let $s \in \mathbb{S}^{\kappa}$, let $M$ and $\varepsilon$ be positive real numbers and let $\dot{A}_{k}$ be names for the elements of $\wp(\mathbb{N}) \cap V$ such that

$$
s \Vdash\left\{\begin{array}{l}
\forall k \in \mathbb{N}, \quad\left\|\dot{\mu}_{k}\right\| \leq \check{M}, \\
\forall k, k^{\prime} \in \mathbb{N}, \quad k \neq k^{\prime}, \quad \dot{A}_{k} \cap \dot{A}_{k^{\prime}}=\emptyset, \\
\forall k \in \mathbb{N}, \quad\left|\dot{\mu}_{k}\left(\dot{A}_{k}\right)\right| \geq \check{\varepsilon} .
\end{array}\right.
$$

By induction, we will construct a sequence $\left(s_{N}\right)_{N \in \mathbb{N}}$ with $s_{N+1} \leq_{F_{N}, N} s_{N}$ where $F_{N}=\left\{\alpha_{i}^{k}: i, k<N\right\}$ and $\operatorname{supp}\left(s_{N}\right)=\left\{\alpha_{k}^{N}: k \in \mathbb{N}\right\}$, a pairwise disjoint sequence $\left(a_{N}\right)_{N \in \mathbb{N}}$ in $\wp(\mathbb{N})$, a pairwise disjoint sequence $\left(E_{N}\right)_{N \in \mathbb{N}}$ of finite subsets of $\mathbb{N}$, sequences of names $\left(\dot{A}_{k}^{N}\right)_{k \in \mathbb{N}, N \in \mathbb{N}}$ for subsets of $\mathbb{N}$ and a sequence of names $\left(\dot{X}_{N}\right)_{N \in \mathbb{N}}$ for subsets of $\mathbb{N}$ such that $s_{N+1}$ forces that

(1) for all $k \in \mathbb{N}, \dot{A}_{k}^{N+1}=\dot{A}_{k}^{N} \backslash \check{a}_{N+1}$, and so, for all $k, k^{\prime} \in \mathbb{N}$ if $k \neq k^{\prime}$, then $\dot{A}_{k}^{N+1} \cap \dot{A}_{k^{\prime}}^{N+1}=\emptyset$;

(2) $\dot{X}_{N+1} \subseteq \dot{X}_{N}$ and $\dot{X}_{N+1}$ is infinite;

(3) for all $k \in \dot{X}_{N+1},\left|\dot{\mu}_{k}\right|\left(\check{a}_{N+1}\right) \leq \check{\delta}_{N}$, and so, for all $k \in \dot{X}_{N+1},\left|\dot{\mu}_{k}\left(\dot{A}_{k}^{N+1}\right)\right| \geq$ $\check{\varepsilon}_{N}-\check{\delta}_{N}$

(4) for all $k \in \bigcup_{0 \leq i \leq N} \check{E}_{i},\left|\dot{\mu}_{k}\right|\left(\check{a}_{N+1}\right) \leq \check{\delta}_{N}$;

(5) there is $k \in \check{E}_{N+1}$ such that $\left|\dot{\mu}_{k}\left(\check{a}_{N+1}\right)\right| \geq \check{\varepsilon}_{N}-\check{\delta}_{N}$;

(6) $\check{E}_{N+1} \subseteq \dot{X}_{N} \backslash \dot{X}_{N+1}$

where $\varepsilon_{0}=\varepsilon, \delta_{0}=\frac{\varepsilon}{2^{3}}$ and for each $N \in \mathbb{N}, \varepsilon_{N+1}=\varepsilon_{N}-\delta_{N}$ and $\delta_{N+1}=\frac{\delta_{N}}{2}$.

For the construction, let $s_{0}=s, E_{0}=\emptyset, \dot{A}_{k}^{0}=\dot{A}_{k}, \dot{X}_{0}=\check{\mathbb{N}}$ and note that we have the hypothesis of Proposition 2.4 .

Now suppose we already have $s_{0}, \ldots, s_{N}, E_{0}, \ldots, E_{N},\left(\dot{A}_{k}^{N}\right)_{k \in \mathbb{N}}, \dot{X}_{0}, \ldots, \dot{X}_{N}$, and $a_{1}, \ldots, a_{N}$ as we want. Note that (1),(2),(3) and (6) guarantee that the hypothesis of Proposition 2.4 is satisfied. Then there are $s_{N+1} \leq_{F_{N}, N} s_{N}, a_{N+1} \subseteq \mathbb{N}, E_{N+1} \subseteq$ $\mathbb{N}$, a sequence of names $\left(\dot{A}_{k}^{N+1}\right)_{k \in \mathbb{N}}$ for subsets of $\mathbb{N}$ and a name $\dot{X}_{N+1}$ for a subset of $\mathbb{N}$ satisfying (1)-(6). This concludes the construction of the sequences.

Then, by Lemma 1.6 of [1] there is $s^{*} \in \mathbb{S}^{\kappa}$ such that $s^{*} \leq s_{N}$ for all $N \in \mathbb{N}$. We have that (1) guarantees that $\left(a_{N}\right)_{N \in \mathbb{N}}$ are pairwise disjoint. Moreover, (4) guarantees that

$$
s^{*} \Vdash \forall N \in \mathbb{N} \text {, if } i<N \text { and } k \in \check{E}_{i} \text {, then }\left|\dot{\mu}_{k}\right|\left(\check{a}_{N}\right) \leq \frac{\check{\varepsilon}}{2^{N+3}} .
$$

On the other hand, using (3) and (6) we conclude that

$$
s^{*} \Vdash \forall N \in \mathbb{N} \text {, if } i>N \text { and } k \in \check{E}_{i} \text {, then }\left|\dot{\mu}_{k}\right|\left(\check{a}_{N}\right) \leq \frac{\check{\varepsilon}}{2^{N+3}} \text {. }
$$

Finally we have that (5) guarantees that

$$
s^{*} \Vdash \forall N \in \mathbb{N}, \exists k \in \check{E}_{N},\left|\dot{\mu}_{k}\left(\check{a}_{N}\right)\right| \geq \frac{3 \check{\varepsilon}}{4} .
$$

Let $\left(K_{\alpha}\right)_{\alpha<\omega_{1}} \subseteq \wp(\mathbb{N})$ be an almost disjoint family. For each $\alpha<\omega_{1}$ we have that

$$
s^{*} \Vdash \bigvee_{N \in \check{K}_{\alpha}} \check{a}_{N} \in \wp(\mathbb{N}) \cap V .
$$

By Theorem 1.11 of [1], $s^{*}$ forces that $\left(\check{K}_{\alpha}\right)_{\alpha<\check{\omega}_{1}}$ is an almost disjoint family of subsets of $\mathbb{N}$ and $\check{\omega}_{1}=\omega_{1}$. By Lemma 2.1 applied in $V[G]$, we obtain $s^{* *} \leq s^{*}$ and 
$\alpha \in \omega_{1}$ such that

$$
s^{* *} \Vdash \forall k \in \mathbb{N} \quad \dot{\mu}_{k}\left(\bigvee_{N \in \check{K}_{\alpha}} \check{a}_{N}\right)=\sum_{N \in \check{K}_{\alpha}} \dot{\mu}_{k}\left(\check{a}_{N}\right) .
$$

Take $a=\bigvee_{N \in K_{\alpha}} a_{N}$ and let us see that in $V[G]$, if $\delta=\frac{\varepsilon}{4}$, then there are infinitely many $n \in \mathbb{N}$ such that $\left|\mu_{n}(a)\right| \geq 2 \delta$ and infinitely many $l \in \mathbb{N}$ such that $\left|\mu_{l}(a)\right| \leq \delta$. If $i \in K_{\alpha}$, using (9), (10) and (11), we have that $s^{* *}$ forces that there is $k \in \check{E}_{i}$ such that

$$
\left|\dot{\mu}_{k}(\check{a})\right|=\left|\sum_{N \in \check{K}_{\alpha}} \dot{\mu}_{k}\left(\check{a}_{N}\right)\right| \geq\left|\dot{\mu}_{k}\left(\check{a}_{i}\right)\right|-\sum_{N \in \check{K}_{\alpha} \backslash\{i\}}\left|\dot{\mu}_{k}\right|\left(\check{a}_{N}\right) \geq \frac{3 \check{\varepsilon}}{4}-\frac{\check{\varepsilon}}{4}=2 \check{\delta} .
$$

On the other hand, if $i \notin K_{\alpha}$, then

$$
s^{* *} \Vdash \forall k \in \check{E}_{i} \quad\left|\dot{\mu}_{k}(\check{a})\right|=\left|\sum_{N \in \check{K}_{\alpha}} \dot{\mu}_{k}\left(\check{a}_{N}\right)\right| \leq \sum_{N \in \check{K}_{\alpha}} \frac{\check{\varepsilon}}{2^{N+3}} \leq \frac{\check{\varepsilon}}{4}=\check{\delta} .
$$

Since $a \in \wp(\mathbb{N}) \cap V$ which is identified with $C l o p(K)$, we have that $\chi_{a} \in C(K)$ and therefore $\left(\mu_{k}\right)_{k \in \mathbb{N}}$ does not converge weakly ${ }^{*}$, contradicting our hypothesis and concluding the proof.

\section{REFERENCES}

[1] J. E. Baumgartner. Sacks forcing and the total failure of Martin's axiom. Topology Appl., 19(3):211-225, 1985. MR0794485 (87a:03086)

[2] K. Ciesielski and J. Pawlikowski. The covering property axiom, CPA: a combinatorial core of the iterated perfect set model, volume 164 of Cambridge Tracts in Mathematics. Cambridge University Press, Cambridge, 2004.

[3] H. G. Dales and W. H. Woodin. An introduction to independence for analysts, volume 115 of London Mathematical Society Lecture Note Series. Cambridge University Press, Cambridge, 1987. MR0942216 (90d:03101)

[4] J. Diestel. Grothendieck spaces and vector measures. In Vector and operator valued measures and applications (Proc. Sympos., Alta, Utah, 1972), pages 97-108. Academic Press, New York, 1973. MR0338774 (49:3538)

[5] J. Diestel. Sequences and series in Banach spaces, volume 92 of Graduate Texts in Mathematics. Springer-Verlag, New York, 1984. MR0737004 (85i:46020)

[6] D. H. Fremlin. Consequences of Martin's axiom, volume 84 of Cambridge Tracts in Mathematics. Cambridge University Press, Cambridge, 1984. MR0780933 (86i:03001)

[7] F. J. Freniche. The Vitali-Hahn-Saks theorem for Boolean algebras with the subsequential interpolation property. Proc. Amer. Math. Soc., 92(3):362-366, 1984. MR0759653 (86e:28008)

[8] R. Haydon. A nonreflexive Grothendieck space that does not contain $l_{\infty}$. Israel J. Math., 40(1):65-73, 1981. MR0636907 (83a:46028)

[9] R. Haydon, M. Levy, and E. Odell. On sequences without weak* convergent convex block subsequences. Proc. Amer. Math. Soc., 100(1):94-98, 1987. MR0883407 (89d:46014)

[10] W. Just and P. Koszmider. Remarks on cofinalities and homomorphism types of Boolean algebras. Algebra Universalis, 28(1):138-149, 1991. MR1083827 (92d:03038)

[11] V. Mascioni. Topics in the theory of complemented subspaces in Banach spaces. Exposition. Math., 7(1):3-47, 1989. MR0982154 (90a:46035)

[12] H. P. Rosenthal. On relatively disjoint families of measures, with some applications to Banach space theory. Studia Math., 37:13-36, 1970. MR0270122 (42:5015)

[13] W. Schachermayer. On some classical measure-theoretic theorems for non-sigma-complete Boolean algebras. Dissertationes Math. (Rozprawy Mat.), 214, 1982. MR0673286|(84d:28015)

[14] Z. Semadeni. Banach spaces of continuous functions. Vol. I. PWN-Polish Scientific Publishers, Warsaw, 1971. Monografie Matematyczne, Tom 55. MR0296671 (45:5730) 
[15] J. Steprāns. Sums of Darboux and continuous functions. Fund. Math., 146(2):107-120, 1995. MR:1314977 (95m:26005)

[16] M. Talagrand. Un nouveau $C(K)$ qui possède la propriété de Grothendieck. Israel J. Math., 37(1-2):181-191, 1980. MR0599313 (82g:46029)

Departamento de Matemática, Instituto de Matemática e Estatística, Universidade de São Paulo, Caixa Postal 66281, CEP: 05315-970, São Paulo, Brasil

E-mail address: kika@ime.usp.br 